\title{
BMJ Open Reperfusion strategy and in-hospital outcomes for ST elevation myocardial infarction in secondary and tertiary hospitals in predominantly rural central China: a multicentre, prospective and observational study
}

You Zhang, ${ }^{1,2,3}$ Shan Wang, ${ }^{1,2,3}$ Qianqian Cheng, ${ }^{1,3}$ Junhui Zhang, ${ }^{1,2,3}$ Datun Qi, ${ }^{1,3}$ Xianpei Wang, ${ }^{1,3}$ Zhongyu Zhu, ${ }^{1,3}$ Muwei Li, ${ }^{1,3}$ Dayi Hu, ${ }^{2,4}$ Chuanyu Gao (D) , , ${ }^{1,3}$ Henan STEMI registry Study Group

To cite: Zhang Y, Wang S, Cheng $Q$, et al. Reperfusion strategy and in-hospital outcomes for ST elevation myocardial infarction in secondary and tertiary hospitals in predominantly rural central China: a multicentre, prospective and observational study. BMJ Open 2021;11:e053510. doi:10.1136/ bmjopen-2021-053510

- Prepublication history for this paper is available online. To view these files, please visit the journal online (http://dx.doi org/10.1136/bmjopen-2021053510).

YZ and SW contributed equally.

Received 14 May 2021

Accepted 24 November 2021

Check for updates

(c) Author(s) (or their employer(s)) 2021. Re-use permitted under CC BY-NC. No commercial re-use. See rights and permissions. Published by BMJ.

For numbered affiliations see end of article.

Correspondence to Professor Chuanyu Gao; gaocy6802@zzu.edu.cn

\section{ABSTRACT}

Objectives To assess differences in reperfusion treatment and outcomes between secondary and tertiary hospitals in predominantly rural central China.

Design Multicentre, prospective and observational study. Setting Sixty-six (50 secondary and 16 tertiary) hospitals in Henan province, central China.

Participants Patients with ST elevation myocardial infarction (STEMI) within 30 days of symptom onset during 2016-2018.

Primary outcome measures In-hospital mortality, and in-hospital death or treatment withdrawal.

Results Among 5063 patients of STEMI, 2553 were treated at secondary hospitals. Reperfusion $(82.0 \%$ vs $73.0 \%, \mathrm{p}<0.001)$ including fibrinolytic therapy $(70.3 \%$ vs $4.4 \%, p<0.001$ ) were more preformed, whereas primary percutaneous coronary intervention ( $11.7 \%$ vs $68.6 \%$, $\mathrm{p}<0.001)$ were less frequent at secondary hospitals. In secondary hospitals, $53 \%$ received fibrinolytic therapy 3 hours after onset, and $5.8 \%$ underwent coronary angiography 2-24 hours after fibrinolysis. Secondary hospitals had a shorter onset-to-first-medical-contact time ( $176 \mathrm{~min}$ vs $270 \mathrm{~min}, \mathrm{p}<0.001)$. Adjusted in-hospital mortality (adjusted OR $1.23,95 \% \mathrm{Cl} 0.89$ to $1.70, \mathrm{p}=0.210$ ) and in-hospital death or treatment withdrawal (adjusted OR $1.18,95 \% \mathrm{Cl} 0.82$ to $1.70, \mathrm{p}=0.361$ ) were similar between secondary and tertiary hospitals.

Conclusions With fibrinolytic therapy as the main reperfusion strategy, the reperfusion rate was higher in secondary hospitals, whereas in-hospital outcomes were similar compared with tertiary hospitals. Public awareness, capacity of primary and secondary care institutes to treat STEMI, and establishment of deeper cooperation among different-level healthcare institutes need to further improve.

Trial registration number NCT02641262.

\section{INTRODUCTION}

With the effect of changing lifestyles and an ageing population, the prevalence of cardiovascular disease is continuously rising
Strengths and limitations of this study

- The registry enrolled 5063 patients from 66 hospitals in Henan, covering 16 out of 18 prefectures in Henan province, which is the largest multicentre, prospective ST elevation myocardial infarction (STEMI) registry in predominantly rural central China.

- Broad inclusion of secondary hospitals will allow for the exploration of STEMI in predominantly rural central China.

- The centres in this study were not randomly selected but volunteered to participate. Therefore, the practice patterns at such centres might not necessarily represent practice at all hospitals in Henan, central China.

- Data collection burden for investigators may be the greatest barrier to the registry that may lead to some enrolment bias.

- A multivariable model was used to adjust for demographic and clinical covariates, but residual measured and unmeasured confounding cannot be excluded.

in China, with estimates of 290 million individuals being affected by the disease. ${ }^{12}$ Cardiovascular diseases including ST elevation myocardial infarction (STEMI) remain the lead cause of death in China and worldwide, ${ }^{2-4}$ and cardiovascular mortality in rural China has been higher than that of urban areas since 2009. ${ }^{2}$ Thrombolysis and primary percutaneous coronary intervention (PCI) have improved the prognosis of patients with STEMI. ${ }^{5-7}$ However, large gap existed between clinical practice and guideline recommendation in China. ${ }^{8}$ Morever, significant geographical variations and hospital-level 
differences persist in process of care and outcomes for patients with STEMI in China. ${ }^{1011}$

There are limited data available on reperfusion treatment and in-hospital outcomes in rural areas of China, especially in predominantly rural central China. A national study showed that central China had the lowest reperfusion rate among the three areas of China (western, central, or eastern), but the best mortality outcomes. ${ }^{10}$ In rural areas of China, secondary hospitals are often firstvisited hospitals for patients of STEMI. However, the proportion of secondary hospitals included in national prospective multicentre STEMI clinical trials was very low, approximately $26 \%$ in the China Acute Myocardial Infarction Registry, ${ }^{12}$ and $22 \%$ in the ongoing Improving Care for Cardiovascular Disease in China-Acute Coronary Syndrome project. ${ }^{13}$

Therefore, we used data from the Henan STEMI registry to assess differences in clinical characteristics, reperfusion therapy, time delays and in-hospital outcomes between secondary and tertiary hospitals in Henan, predominantly rural areas of central China.

\section{METHODS}

\section{Study design}

The design of Henan STEMI registry has been described previously. ${ }^{14}$ Briefly, it is a multicentre, prospective, and observational study aimed to evaluate the characteristics, management and outcomes of patients with STEMI as seen in routine clinical practice. Only secondary and tertiary hospitals are reperfusion capable, which are located in three levels of Chinese local governmentprovince, prefecture and county. Therefore, this registry included 66 (50 secondary and 16 tertiary) hospitals from 15 prefectures of Henan.

\section{Participants}

Patients with a primary diagnosis of STEMI admitted within 30 days of symptom onset were consecutively enrolled between September 2016 and August 2018. STEMI was defined in accordance with the universal definition of myocardial infarction (MI), ${ }^{15}$ specifically as persistent ST-segment elevation $(\geq 0.1 \mathrm{mV}$ at J points) in two or more contiguous leads or new onset of left bundle branch block. Furthermore, according to the classification of MI, types $4 \mathrm{a}$ and type 5 are excluded from this registry.

Among 5479 patients from 66 hospitals, we excluded 137 cases who did not meet the study criteria to create the study sample of 5342 patients with STEMI (2562 in secondary hospitals, and 2780 in tertiary hospitals). For the main analysis of in-hospital treatments and outcomes, we further excluded 279 cases with prior reperfusion because it may influence the doctor's treatment choices.

Patient and public involvement

No patient involved.

\section{Definitions and data collection}

Clinical data are collected by trained investigators via a secure, password protected, web-based data collection platform (Henan STEMI registry platform, Zhao Rui Corporation, Zhengzhou). Data elements collected include patient demographics, risk factors, medical histories, prehospital information, in-hospital treatment, reperfusion strategies and clinical events. To ensure the accuracy and completeness of data, besides universal definition of STEMI, face-to-face training workshops, and use of a standardised online reporting tool with automatic checks for invalid values, we check the consecutiveness of all cases, and monitor $53.8 \%$ of reported cases for accuracy against medical records for onsite quality control.

Hypertension was defined as having a history of hypertension, or receiving antihypertensive therapy. Dyslipidaemia was defined according to the guideline for prevention and treatment of adult dyslipidaemia in China as total cholesterol $\geq 5.2 \mathrm{mmol} / \mathrm{L}$, low density lipoprotein $\geq 3.4 \mathrm{mmol} / \mathrm{L}$, or high density lipoprotein $\leq 1.0 \mathrm{mmol} / \mathrm{L}$. ${ }^{16}$ Diabetes mellitus was defined as having a previous diagnosis of diabetes mellitus, or haemoglobin A1c level $\geq 6.5 \%$. Current smoking was defined as smoking within the preceding year. A history of coronary heart disease was specified if patients had a clinical history of myocardial infarction or underwent PCI or coronary artery bypass grafting before the current hospitalisation. The wall location of the MI was determined by ECG.

Patients eligible for reperfusion were defined as those with primary diagnosis of STEMI, and admitted within 12 hours after symptom onset. We also recorded contraindications for fibrinolysis. Specific thrombolytic agents refer to a class of thrombolytic agents that selectively activate fibrin-binding plasminogen in thrombus, and have little effect on systemic fibrinolytic activity, low risk of bleeding and high recanalisation rate. Among them, alteplase, prourokinase and reteplase were used in our study. The success of thrombolysis was assessed according to indirect measures of vascular recanalisation (clinical judgement criteria), including significant relief of chest pain, ST segment resolution $\geq 50 \%$, occurrence of reperfusion arrhythmia, and early peak value of myocardial necrosis markers. ${ }^{17}$

Treatment delays were considered from symptom onsetto-first medical contact (FMC, defined as time of diagnostic ECG), FMC-to-fibrinolysis (from FMC to initiation of thrombolytic therapy), and FMC-to-PCI (from FMC to wire passage into the culprit artery). Door-in door-out time was defined as time from admission to discharge at non-primary PCI centres among patients receiving transfer PCI.

The primary outcome was in-hospital all-cause mortality. Because treatment withdrawal is common in China (most patients are reluctant to die in hospital), we used in-hospital all death or treatment withdrawal as the main in-hospital outcome. We compared in-hospital mortality, in-hospital all death or treatment withdrawal, and in-hospital main adverse cardiovascular and cerebrovascular 
events (MACCE, death or treatment withdrawal, congestive heart failure, reinfarction and ischaemic stroke) among different-level hospitals.

\section{Statistical analysis}

Categorical variables are presented as number and percentage, and were compared using the $\chi^{2}$ or Fisher exact tests among different-level hospitals. Continuous variables are presented as median (IQR) and differences between secondary and tertiary hospitals were compared using the Mann-Whitney $\mathrm{U}$ test.

Information about smoking was missing for $23(0.5 \%)$ patients, and we used the multiple imputation method to impute the variable. ${ }^{18}$ For the missing data on other risk factors or medical history $(\leq 0.7 \%)$, we imputed sample medians. Data on postfibrinolysis angiography and DIDO time were missing in $229(16.1 \%)$ and $47(10.4 \%)$ patients, respectively. No other data were missing.

To examine the association between hospital class and in-hospital outcomes, we used generalised linear mixed model to account for clustering of patients within hospitals. The dependent variables were in-hospital death; in-hospital death or treatment withdrawal; and in-hospital MACCE, respectively. The independent variables were patients' demographics (age and sex); risk factors or medical history (hypertension, diabetes, current smoker, previous coronary heart disease and previous stroke); clinical characteristics at admission (cardiac arrest, heart rate, systolic blood pressure, anterior MI, cardiogenic shock and prehospital ECG); and symptom onset to FMC time. We transformed continuous variables (age, heart rate, systolic blood pressure and symptom onset to FMC time) into categorical variables according to clinically meaningful cut-off values (tables 1 and 2).

We used two methods for sensitivity analysis. First, we used 7 day outcomes instead of the original outcomes. Second, we excluded patients who were transferred out or discharged within 24 hours because they probably left against medical advice and there was very little time for treatment. For these models we report ORs with 95\% CIs for secondary hospital versus tertiary hospital. Two-sided $\mathrm{p}$ values $<0.05$ were considered statistically significant. Statistical analyses were performed with SAS 9.4 (SAS Institute).

\section{RESULTS}

\section{Baseline characteristics}

A total of 5063 patients were included in the final analysis: 2553 and 2510 came from secondary and tertiary hospitals, respectively (figure 1). Compared with patients with STEMI in tertiary hospitals, those in secondary centres were significantly older, with lower proportions of men, hypertension, dyslipidaemia, diabetes, smoking, and prior stroke. At admission, patients in secondary hospitals were less likely to be transferred from another hospital, to be admitted by ambulance, to bypass the emergency room to catheter lab, and to have prehospital
ECG, typical chest pain, and cardiac arrest, and instead, they had a higher prevalence of cardiogenic shock (table 1).

\section{Treatment delays}

Patients in tertiary hospitals had longer onset-to-FMC, FMC-to-fibrinolysis, onset-to-fibrinolysis and onset-to-PCI time than those in secondary hospitals. First ECG was higher in secondary hospitals. In secondary hospitals, more than half patients received fibrinolysis after 3 hours of symptom onset, and $29.7 \%$ of fibrinolysis was performed in $\leq 30 \mathrm{~min}$, which was higher than that of tertiary hospitals. For patients transferred to tertiary hospitals, few patients $(14.2 \%)$ achieved a DIDO of $\leq 30 \mathrm{~min}$ in tertiary hospitals (table 2).

\section{Reperfusion treatment}

Compared with tertiary hospitals, patients in secondary centres had a significantly higher proportion of reperfusion $(82.0 \%$ vs $73.0 \%, \mathrm{p}<0.001)$ and fibrinolytic therapy ( $70.3 \%$ vs $4.4 \%, \mathrm{p}<0.001)$, but lower utilisation rate of primary PCI $(11.7 \%$ vs $68.6 \%, \mathrm{p}<0.001)$. Of a total of 356 patients hospitalised within 12-24 hour of symptom onset, 83 received reperfusion. There was no difference in the reperfusion rate between different levels of hospitals. In secondary hospitals, $26(15.6 \%)$ patients underwent fibrinolytic therapy between 12 and 24 hours after onset of symptoms. One more patient admitted between 24 and 48 hours of symptom onset in tertiary hospital was treated with primary PCI. Among 1517 patients treated with primary PCI, tertiary hospitals had higher utilisation of transfer PCI and lower proportion of stents. Among the patients who received fibrinolytic therapy in secondary hospitals, $95.1 \%$ were treated with specific thrombolytic agents, but $17.2 \%$ failed, of which $5.6 \%$ were referred to rescue PCI. And $5.8 \%$ underwent coronary angiography 2-24hours after fibrinolysis (table 3).

\section{In-hospital outcomes}

Before adjustment, patients in secondary hospitals has higher in-hospital mortality $(4.6 \%$ vs $3.2 \%, \mathrm{p}=0.013)$ or in-hospital death or treatment withdrawal $(8.6 \%$ vs $6.9 \%, \mathrm{p}=0.025)$. However, there was no difference in in-hospital death, or in-hospital death or treatment withdrawal between secondary and tertiary hospitals after adjusting for sociodemographic variables, risk factors, medical history, clinical characteristics at admission and symptom onset to FMC time. Similarly, the adjusted risk of MACCE was similar between the two classes of hospitals (figure 2). Adjusted outcomes calculated with a 7-day timeframe were similar to those of the primary analyses using the entire hospital stay (figure 2). A sensitivity analysis compared the results of the entire cohort with those of the population excluding patients who transferred out or discharged within 24 hours. The results of these two analyses did not differ (figure 2). 
Open access

Table 1 Baseline characteristics of patients with STEMI

\begin{tabular}{|c|c|c|c|}
\hline & $\begin{array}{l}\text { Secondary hospital } \\
(n=2553)\end{array}$ & $\begin{array}{l}\text { Tertiary hospital } \\
(\mathrm{n}=2510)\end{array}$ & $P$ value \\
\hline Age, years & $64.2(54.0-72.0)$ & $62.0(51.4-70.0)$ & $<0.001$ \\
\hline$\geq 75$ years & $483(18.9)$ & $371(14.8)$ & $<0.001$ \\
\hline Women & $693(27.1)$ & $541(21.6)$ & $<0.001$ \\
\hline \multicolumn{4}{|l|}{ Risk factors } \\
\hline Hypertension & $1029(40.3)$ & $1204(48.0)$ & $<0.001$ \\
\hline Dyslipidaemia & $1297(50.8)$ & $1424(56.7)$ & $<0.001$ \\
\hline Diabetes & $382(15.0)$ & $503(20.0)$ & $<0.001$ \\
\hline Current smoker & $870(34.1)$ & $1133(45.1)$ & $<0.001$ \\
\hline \multicolumn{4}{|l|}{ Medical history } \\
\hline Stroke & $296(11.6)$ & $360(14.3)$ & 0.004 \\
\hline Ischaemic & $275(10.8)$ & $336(13.4)$ & 0.004 \\
\hline Haemorrhagic & $26(1.0)$ & $32(1.3)$ & 0.39 \\
\hline Coronary heart disease & $159(6.2)$ & $155(6.2)$ & 0.94 \\
\hline Myocardial infarction & $127(5.0)$ & $131(5.2)$ & 0.69 \\
\hline Percutaneous coronary intervention & $73(2.9)$ & 89 (3.6) & 0.17 \\
\hline Coronary artery bypass graft & $3(0.1)$ & $4(0.2)$ & 0.72 \\
\hline \multicolumn{4}{|l|}{ Clinical characteristic } \\
\hline Transferred in & $79(3.1)$ & $1063(42.4)$ & $<0.001$ \\
\hline Hospital approaching method & & & $<0.001$ \\
\hline Self-transport & $1984(77.7)$ & $1630(64.9)$ & \\
\hline By ambulance & $530(20.8)$ & $866(34.5)$ & \\
\hline On site & $39(1.5)$ & $14(0.6)$ & \\
\hline Prehospital ECG & $340(13.3)$ & $710(28.3)$ & $<0.001$ \\
\hline Bypassing the ER & $20(0.8)$ & $114(4.5)$ & $<0.001$ \\
\hline Myocardial ischaemia symptoms & & & $<0.001$ \\
\hline Typical & $2038(79.8)$ & 2215 (88.3) & \\
\hline Atypical & $508(19.9)$ & $284(11.3)$ & \\
\hline No symptom & $7(0.3)$ & $11(0.4)$ & \\
\hline Cardiac arrest & $52(2.0)$ & $102(4.1)$ & $<0.001$ \\
\hline Cardiogenic shock & $192(7.5)$ & $125(5.0)$ & $<0.001$ \\
\hline Anterior myocardial infarction & $1452(56.9)$ & $1423(56.7)$ & 0.90 \\
\hline LBBB & $22(0.9)$ & $20(0.8)$ & 0.80 \\
\hline Heart rate (beats/min) & & & $<0.001$ \\
\hline$<50$ & $149(5.8)$ & $80(3.2)$ & \\
\hline $50-109$ & $2278(89.2)$ & $2277(90.7)$ & \\
\hline$\geq 110$ & $126(4.9)$ & $153(6.1)$ & \\
\hline Systolic blood pressure $(\mathrm{mm} \mathrm{Hg})$ & & & $<0.001$ \\
\hline$<90$ & $151(5.9)$ & $95(3.8)$ & \\
\hline $90-139$ & $1374(53.8)$ & $1628(64.9)$ & \\
\hline$\geq 140$ & $1028(40.3)$ & 787 (31.4) & \\
\hline Length of stay, days & $10(6-13)$ & $11(8-14)$ & $<0.001$ \\
\hline
\end{tabular}

Data are presented as median (IQR) or $\mathrm{n}(\%)$.

ECG, electrocardiogram; ER, emergency room; LBBB, left bundle branch block; STEMI, ST elevation myocardial infarction. 
Table 2 Treatment delays among patients with STEMI

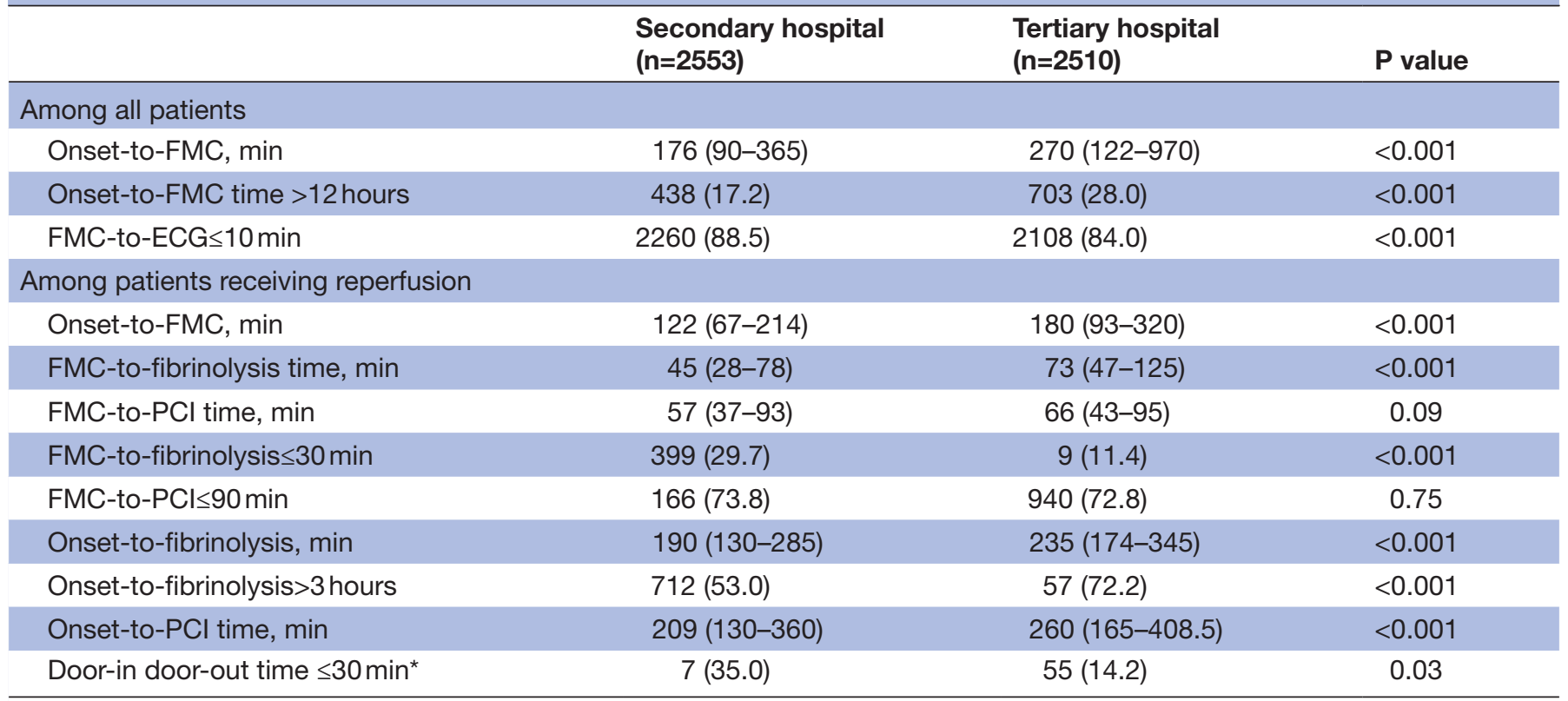

Data are presented as median (IQR) or $\mathrm{n}(\%)$.

${ }^{*}$ Data were missing in $10.4 \%$ of the records.

FMC, first medical contact; PCI, percutaneous coronary intervention; STEMI, ST elevation myocardial infarction.

\section{DISCUSSION}

Based on data collected in the multicentre, prospective Henan STEMI registry on real-world reperfusion, treatment delays and in-hospital outcomes of patients with STEMI in predominantly rural central China, we here report that different practice patterns exist between hospital tiers, with thrombolysis mainly used in secondary hospitals and primary intervention in tertiary hospitals. The reperfusion rate was higher in secondary hospitals, whereas in-hospital outcomes were similar between secondary and tertiary hospitals. There is still a large gap between reperfusion practices and those recommended by guidelines, such as delayed thrombolysis without further angiographic assessment and prolonged prehospital delay.

Our results showed substantial gaps in the use of thrombolytic therapy compared with guidelines. Similar to other developing countries, fibrinolytic therapy remains the main reperfusion therapy in secondary hospitals. ${ }^{19} 20$ With a vast territory, and limited by medical condition, geographical location and techniques, early fibrinolysis and/or transfer PCI strategy should be the priority in

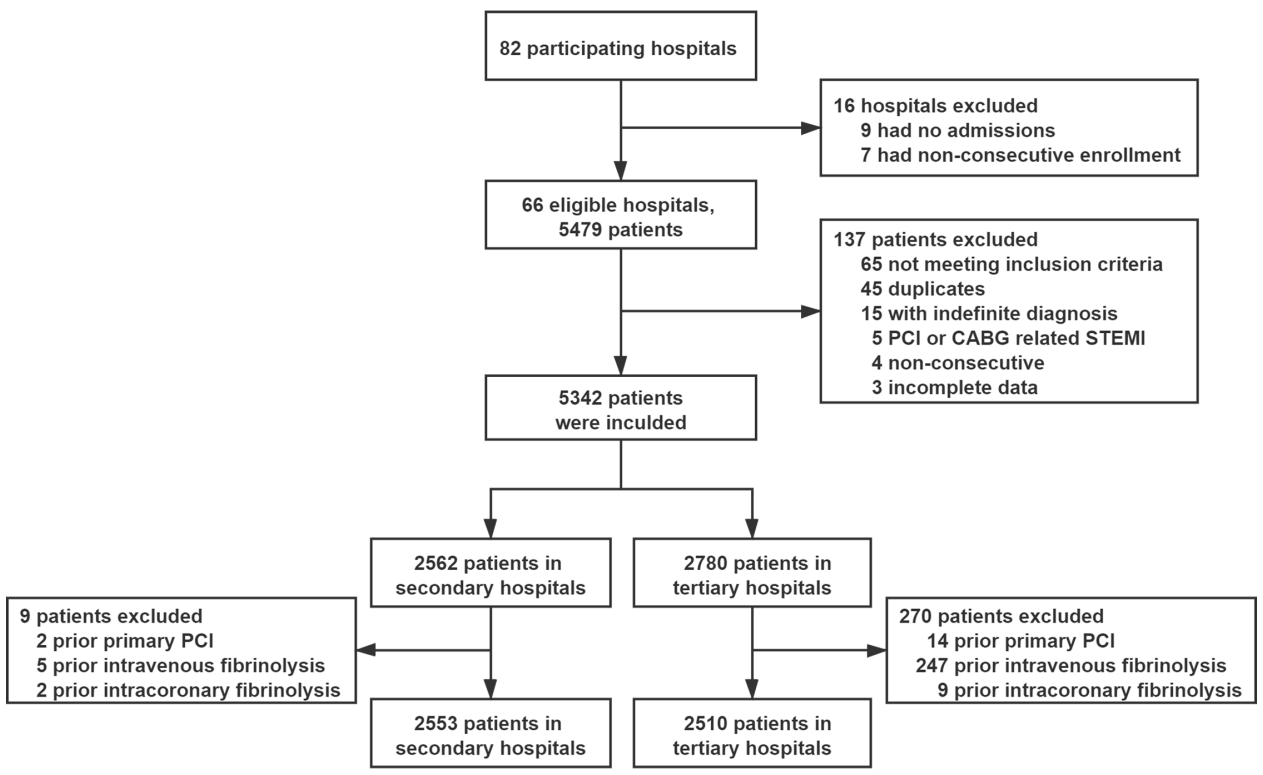

Figure 1 Study profile. CABG, coronary artery bypass grafting; PCI, percutaneous coronary intervention; STEMI, ST elevation myocardial infarction. 
Table 3 Reperfusion therapy among patients with STEMI

\begin{tabular}{|c|c|c|c|}
\hline & $\begin{array}{l}\text { Secondary hospital } \\
(\mathrm{n}=2553)\end{array}$ & $\begin{array}{l}\text { Tertiary hospital } \\
(n=2510)\end{array}$ & $P$ value \\
\hline \multicolumn{4}{|l|}{ Among all patients } \\
\hline Reperfused & $1569(61.5)$ & $1371(54.6)$ & $<0.001$ \\
\hline Fibrinolysis & $1344(52.6)$ & $79(3.1)$ & $<0.001$ \\
\hline Primary PCl & $225(8.8)$ & $1292(51.5)$ & $<0.001$ \\
\hline STEMI eligible for reperfusion & $1875(73.4)$ & $1807(72.0)$ & $<0.001$ \\
\hline \multicolumn{4}{|l|}{ Among eligible } \\
\hline Reperfused & $1538(82.0)$ & $1318(73.0)$ & $<0.001$ \\
\hline Fibrinolysis & $1318(70.3)$ & $79(4.4)$ & $<0.001$ \\
\hline Primary PCl & $220(11.7)$ & $1239(68.6)$ & $<0.001$ \\
\hline \multicolumn{4}{|l|}{ Among all STEMI } \\
\hline Admitted $12-24$ hours & $167(6.5)$ & $189(7.5)$ & 0.17 \\
\hline Reperfused & $31(18.6)$ & $52(27.5)$ & 0.06 \\
\hline Fibrinolysis & $26(15.6)$ & - & - \\
\hline Primary PCl & $5(3.0)$ & $52(27.5)$ & $<0.001$ \\
\hline Among primary $\mathrm{PCl}$ & 225 & 1292 & \\
\hline Transfer PCl & $20(8.9)$ & $434(33.6)$ & $<0.001$ \\
\hline Stent & 203 (90.2) & $1092(84.5)$ & 0.03 \\
\hline Among fibrinolysis & 1344 & 79 & \\
\hline Specific thrombolytic agents & $1278(95.1)$ & $74(93.7)$ & 0.77 \\
\hline Failed fibrinolysis & $231(17.2)$ & $20(25.3)$ & 0.07 \\
\hline Fibrinolysis +rescue PCl & $13(5.6)$ & $2(10.0)$ & 0.76 \\
\hline Coronary angiography $2-24$ hours post fibrinolysis* & $66(5.8)$ & $6(10.0)$ & 0.17 \\
\hline
\end{tabular}

Data are presented as $\mathrm{n}(\%)$.

*Data were missing in $16.1 \%$ of the records.

$\mathrm{PCl}$, percutaneous coronary intervention; STEMI, ST elevation myocardial infarction.

secondary hospitals without PCI capability in Henan. ${ }^{21-24}$ However, there is still much room for improvement in thrombolytic therapy. First, the proportion of early

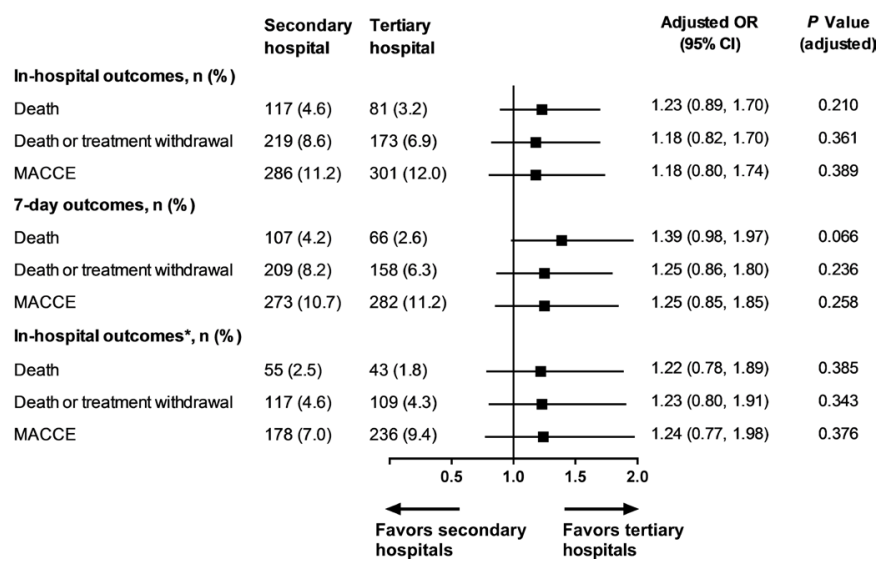

Figure 2 Adjusted in-hospital outcomes for patients with STEMI. STEMI, ST elevation myocardial infarction; MACCE, adverse cardiovascular and cerebrovascular events. MACCE included death or treatment withdrawal, congestive heart failure, reinfarction and ischaemic stroke. *Without patients who were transferred out or discharged within 24 hours. fibrinolytic therapy was low. Our finding showed that more than half the patients received fibrinolytic therapy after 3 hours of symptom onset; $15.6 \%$ of patients in secondary hospitals even received it beyond 12 hours of symptom onset; and only $29.7 \%$ of patients undergoing fibrinolysis within 30 min of FMC. Second, there was no emphasis on timely evaluation with coronary angiography after fibrinolysis. Current guidelines recommend that patients with STEMI should be transferred to a PCI-capable hospital for coronary angiography between 2 and 24 hours after successful fibrinolysis. ${ }^{5-7}$ However, in the present study, only $5.8 \%$ underwent early angiography in accordance with the guidelines in secondary hospitals, which was lower than in other countries. ${ }^{2025}$ Furthermore, only $5.6 \%$ of the patients who failed fibrinolysis underwent rescue PCI. These gaps reflect the insufficient public knowledge of STEMI, ${ }^{26}$ poor ability of primary healthcare to identify and treat STEMI, ${ }^{27}$ inability of most secondary hospitals to provide $24 / 7$ PCI service, ${ }^{28}$ and inadequate medicare payment systems, ${ }^{27}{ }^{29}$ which need further improvement.

Although primary PCI is the mainstay reperfusion method in tertiary hospitals in central China, the proportion of primary PCI is lower compared with developed 
countries. ${ }^{30-33}$ Moreover, our results showed that the early reperfusion rate was lower in tertiary hospitals compared with secondary hospitals, despite tertiary hospitals having more advanced facilities and greater availability of specialists, possibly due to the following reasons. First, prolonged prehospital delay resulted in a lower percentage of patients meeting reperfusion criteria. In our study, prehospital delays were observed in both secondary and tertiary hospitals, especially in tertiary hospitals. The median onset-to-FMC time was nearly greater than 3 hours in both hospitals, significantly higher than that of other studies. ${ }^{2030} 33$ Prehospital delays mainly results from the healthcare system and patients. ${ }^{21} 2634$ Our study found low percentage of ambulance transport and longer DIDO time. Second, patients in secondary hospitals were not timely transferred to tertiary hospitals after thrombolysis. Therefore, it is in urgent need in tertiary hospitals to establish an integrated regional network including the central coordination of emergency medical system, patient referral from primary healthcare facilities to PCI hospitals, interhospital transfer and coordinated clinical pathway in PCI hospitals. ${ }^{26}$ This is just the purpose of the China Chest Pain Center project. ${ }^{35}$ It has been showed that accreditation of chest pain centre is associated with better management and in-hospital outcomes. ${ }^{36}{ }^{37}$ In 2020, the establishment of chest pain care units in primary healthcare institutions has been initiated to improve the ability of primary care physicians to recognise chest pain and STEMI diagnosis. ${ }^{38}$

Our finding showed that in-hospital outcomes were similar in secondary and tertiary hospitals, although the reperfusion use rate was higher in secondary hospitals. Several reasons underlie this situation. First, the main risk factors for death, such as the elderly, women and cardiogenic shock, were more common in secondary hospitals than in tertiary hospitals; Second, most secondary hospitals without PCI capability administered delayed thrombolysis without further angiographic assessment; finally, the first two reasons make tertiary hospitals with PCI capability unable to give full play to the advantages of intervention.

In this study, we identified substantial gaps between reperfusion practices and guidelines in predominantly rural central China, including prolonged prehospital delays, delayed thrombolysis without further angiographic assessment and low PCI use. The main reasons are poor public awareness of STEMI ${ }^{26}$ low quality of primary and secondary healthcare, ${ }^{27}$ poor coordination between primary, secondary and tertiary healthcare institutes and inadequate medicare payment systems. ${ }^{27} 29$ To improve the quality care for STEMI in rural China, it is necessary to further increase public awareness, the capacity of primary and secondary healthcare institutes, and establish deeper cooperation among different-level healthcare institutes, such as establishment of medical alliance, chest pain centre and chest pain care unit.

The Henan STEMI registry is distinguished that it is a large multicentre, prospective regional STEMI registry, including both secondary and tertiary hospitals in predominantly rural central China; our finding will help policy makers and healthcare professionals comprehensively get the knowledge of real-world practice care, treatment delays and outcomes in secondary and tertiary hospitals in central China, and facilitate the translation of study findings to improvement of quality care for STEMI in China and other countries in a similar situation.

\section{Limitations}

Several limitations of our study should be discussed. First, the centres in our study (as for most registries) were not randomly selected but volunteered to participate. Therefore, the practice patterns at such centres might not necessarily represent practice at all hospitals in Henan, central China. However, the geographical diversity (16 of the total 18 prefectures) gives an indication of the usefulness of the data. Second, the data collection burden for investigators may be the greatest barrier to the registry that may lead to some enrolment bias. We have carefully considered each element to limit the burden and have quality control measures in the registry. Third, we used a multivariable model to adjust for demographic and clinical covariates, but residual measured and unmeasured confounding cannot be excluded. However, the sensitivity analysis results confirmed that our results were relatively stable.

\section{CONCLUSION}

Different classed of hospital have different reperfusion methods. Fibrinolytic therapy was the main reperfusion therapy in secondary hospitals, and more than half of the patients had delayed thrombolysis and only a few underwent timely angiographic evaluation. The reperfusion rate was higher in secondary hospitals, whereas in-hospital outcomes were similar between secondary and tertiary hospitals. Improvement of quality of care for STEMI should underscore raising public awareness, enhancing the quality of medical care in primary and secondary healthcare institutes, and establishment of deeper cooperation among different-level healthcare institutes. Our findings provide evidence for policymakers and healthcare professionals in China and other developing countries.

\section{Author affiliations}

${ }^{1}$ Department of Cardiology, Central China Fuwai Hospital of Zhengzhou University, Henan Provincial People's Hospital Heart Center, Zhengzhou, Henan, China

${ }^{2}$ Henan Institute of Cardiovascular Epidemiology, Zhengzhou, Henan, China ${ }^{3}$ Henan Key Laboratory for Prevention and Control of Coronary Heart Disease, Central China Fuwai Hospital of Zhengzhou University, Zhengzhou, Henan, China ${ }^{4}$ Institute of Cardiovascular Disease, Peking University People's Hospital, Beijing, China

Acknowledgements We would like to thank all of the members of Scientific Committee, and Executive Committee for their contribution to the Henan STEMI registry. We also want to thank all of the study investigators and coordinators for their great work, and thank Prof. Xi Li, associate research fellow in National Clinical Research Center for Cardiovascular Diseases, Fuwai Hospital, for his guidance in manuscript preparation. 
Collaborators Henan STEMI registry Study Group: Henan Provincial People's Hospital, Chuanyu Gao; Xinxiang Central Hospital, Zhifang Wang and Shuhong Su; The First Affiliated Hospital of Henan Science and Technology University, Pingshuan Dong and Xuming Yang; The First People's Hospital of Shangqiu, Shengli Li; Xiping County People's Hospital, Hualing Xia and Wuxing Li; Zhumadian Central Hospital, Shuiyin Ding, Baoqiang Bai and Xinyuan Yang; The People's Hospital of Yongcheng, Changming Tian; Xihua County People's Hospital, Jinbo Li and Chuntong Wang; Shangcai County People's Hospital, Yi Yang; The People's Hospital of Gongyi, Yanhui Gao; The Third Affiliated Hospital of Xinxiang Medical College, Haiyan Sun; The Second People's Hospital of Xinxiang, Jixia Yan; The People's Hospital of Xingyang, Heping Li; The First People's Hospital of Lingbao, Wanke Li; Zhecheng County People's Hospital, Zhenfu Zhao; The Second People's Hospital of Nanyang, Huading Zhou; Ningling County People's Hospital, Chuanqian Zhang; Puyang Oil Field General Hospital, Hengliang Wang; Fengqiu County People's Hospital, Dequan Jing; Mianchi County People's Hospital, Suxia Chen; Wen County People's Hospital, Xiaoli Ji; The People's Hospital of Jiaozuo, Haijun Zheng; New area People's Hospital of Luoyang, Fengxian Lin; The First People's Hospital of Xinxiang, Guiye Zhao; Pingyu County People's Hospital, Wei Wei; The People's Hospital of Hebi, Peng Liu and Yanggui Liu; Xiayi County People's Hospital, Xiangyang Cheng; The People's Hospital of Qinyang, Xiaowen Ma; Tongxu County Hospital of Chinese medicine, Chengwen Zhang; Song County People's Hospital, Liangping Wang; The Third People's Hospital of Shangqiu, Changgang Tong; The Second People's Hospital of Pingdingshan, Ling Zhang; The People's Hospital of Yanshi, Huihui Lang; Yudong Hospital of the First Affiliated Hospital of Henan University of Chinese Medicine, Haojie Xu; The Second People's Hospital of Mengjin County, Hengshan Wei and Haitao Wang; The Second People's Hospital of Jiyuan, Ruilu Xue; The People's Hospital of Dengfeng, Hongxu Geng; Minquan County People's Hospital, Yonghong Shi; The First Affiliated Hospital of Henan University, Qiwei Tang; Nanzhao County People's Hospital, Yuchun Li; The Second People's Hospital of Xiayi County, Yonggang Liu; Tongxu County People's Hospital, Dongsheng Wang; Neixiang County People's Hospital, Jianbo Jia; Xuchang County People's Hospital, Xianzhang Li; Zhecheng County Hospital of Chinese Medicine, Xinling Deng; The Sixteenth People's Hospital of Zhengzhou, Qinghua Xu; Nanshi Hospital of Nanyang, Linming Zhao; Yuzhou City Hospital of Chinese Medicine, Hongxia Zhang; Sheqi County People's Hospital, Gang Liu; The Third People's Hospital of Luoyang, Lipeng Li and Suqin Chen; The People's Hospital of Wugang, Keqi Liang; Xinye County People's Hospital, Linwu Zhang; Weishi County People's Hospital, Jiandang Jiang; Runan County People's Hospital, Weitian Xiao; Yichuan County Hospital of Chinese Medicine, Hongwen Zhang; Qi County People's Hospital, Shengke Zhu; Yucheng County People's Hospital, Baofu Liu; Minquan County Hospital of Chinese Medicine, Chengxuan Pan; The Central Hospital of Yima Coal Industry Group CO. LTD, Fengyun Lu; Ye County People's Hospital, Jie Yang; Queshan County People's Hospital, Yaoze Li; Huangchuan County People's Hospital, Zheng Liu; Suiping County People's Hospital, Qingchun Zhang; Fangcheng County People's Hospital, Yintao Qiao; The People's Hospital of Jiaozuo Macun District, Na Sun; The Second People's Hospital of Xichuan County, Lianjie Li.

Contributors CG and YZ conceived and designed the study, had full access to all of the data in the study and takes responsibility for the integrity of the data and the accuracy of the data analysis. YZ wrote the first draft or the article, with further contributions from QC, JZ, DQ, XW, ZZ, ML and DH. YZ, SW and CG provided expert clinical opinion and interpretation of the data. SW did statistical analysis, with support from $\mathrm{YZ}$ and $\mathrm{CG}$. All authors made critical revisions and approved the final version of the article.

Funding This work was supported by the Project of Scientific and Technological Support Plan of Health and Family Planning Commission of Henan Province in 2016 [grant number: 201602210] and Shanghai Tasly Pharmaceutical Co. Ltd [grant number: not applicable].

Disclaimer The funding organisations for this study had no involvement in the design and conduct of the study; collection, management, analysis, and interpretation of the data; preparation, review, or approval of the manuscript; or the decision to submit the manuscript for publication.

Competing interests None declared.

Patient and public involvement Patients and/or the public were not involved in the design, or conduct, or reporting, or dissemination plans of this research.

\section{Patient consent for publication Not applicable.}

Ethics approval The medical ethics committee of Henan Provincial People's Hospital approved the Henan STEMI registry with a waiver for informed consent [No. 2015 (34)].

Provenance and peer review Not commissioned; externally peer reviewed.
Data availability statement Data are available upon reasonable request.

Open access This is an open access article distributed in accordance with the Creative Commons Attribution Non Commercial (CC BY-NC 4.0) license, which permits others to distribute, remix, adapt, build upon this work non-commercially, and license their derivative works on different terms, provided the original work is properly cited, appropriate credit is given, any changes made indicated, and the use is non-commercial. See: http://creativecommons.org/licenses/by-nc/4.0/.

ORCID iD

Chuanyu Gao http://orcid.org/0000-0001-7079-2283

\section{REFERENCES}

1 Cheng X, Yang Y, Schwebel DC, et al. Population ageing and mortality during 1990-2017: A global decomposition analysis. PLoS Med 2020;17:e1003138.

2 The Writing Committee of the Report on Cardiovascular Health and Diseases in China. Report on cardiovascular health and diseases in China 2019: an updated summary. Chinese Circulation Journal 2020;35:833-54.

3 GBD 2017 Causes of Death Collaborators. Global, regional, and national age-sex-specific mortality for 282 causes of death in 195 countries and territories, 1980-2017: a systematic analysis for the global burden of disease study 2017. Lancet 2018;392:1736-88.

4 Liu S, Li Y, Zeng X, et al. Burden of cardiovascular diseases in China, 1990-2016: findings from the 2016 global burden of disease study. JAMA Cardiol 2019;4:342-52.

5 Levine GN, Bates ER, Blankenship JC, et al. 2015 ACC/AHA SCAl focused update on primary percutaneous coronary intervention for patients with ST-elevation myocardial infarction: an update of the 2011 ACCF/AHA/SCAI guideline for percutaneous coronary intervention and the 2013 ACCF/AHA guideline for the management of ST-elevation myocardial infarction. J Am Coll Cardiol 2016;67:1235-50.

6 Ibanez B, James S, Agewall S, et al. 2017 ESC guidelines for the management of acute myocardial infarction in patients presenting with ST-segment elevation: the task force for the management of acute myocardial infarction in patients presenting with ST-segment elevation of the European Society of cardiology (ESC). Eur Heart $J$ 2018;39:119-77.

7 Chinese Society of Cardiology of Chinese Medical Association, Editorial board of Chinese Journal of Cardiology. 2019 Chinese Society of cardiology (CSC) guidelines for the diagnosis andmanagement of patients with ST-segment elevation myocardial infarction. ZhonghuaXinXue Guan Bing ZaZhi 2019;47:766-83.

$8 \mathrm{Li} \mathrm{J}$, Li X, Wang Q, et al. ST-segment elevation myocardial infarction in China from 2001 to 2011 (the China PEACE-Retrospective acute myocardial infarction study): a retrospective analysis of hospital data. Lancet 2015;385:441-51.

9 Song F, Yu M, Yang J, et al. Symptom-onset-to-balloon time, ST-segment resolution and in-hospital mortality in patients with ST-segment elevation myocardial infarction undergoing primary percutaneous coronary intervention in China: from China acute myocardial infarction registry. Am J Cardiol 2016;118:1334-9.

10 Zhong Q, Gao Y, Zheng X, et al. Geographic variation in process and outcomes of care for patients with acute myocardial infarction in China from 2001 to 2015. JAMA Netw Open 2020;3:e2021182.

11 Xu H, Yang Y, Wang C, et al. Association of hospital-level differences in care with outcomes among patients with acute ST-segment elevation myocardial infarction in China. JAMA Netw Open 2020;3:e2021677.

$12 \mathrm{Xu} \mathrm{H}$, Li W, Yang J, et al. The china acute myocardial infarction (CAMI) registry: a national long-term registry-research-education integrated platform for exploring acute myocardial infarction in China. Am Heart J 2016;175:193-201.

13 Hao Y, Liu J, Liu J, et al. Sex differences in in-hospital management and outcomes of patients with acute coronary syndrome. Circulation 2019;139:1776-85.

14 Zhang Y, Wang S, Yang S, et al. Rationale and design of the Henan ST elevation myocardial infarction (STEMI) registry: a regional STEMI project in predominantly rural central China. BMC Cardiovasc Disord 2019;19:271.

15 Thygesen K, Alpert JS, Jaffe AS, et al. Third universal definition of myocardial infarction. Circulation 2012;126:2020-35.

16 The Joint Committee on the Revision of Chinese Guidelines for the Prevention and Treatment of Adult Dyslipidemia. Chinese guidelines on prevention and treatment of dyslipidemia in adults (revised in 2016). ZhonghuaXinXue Guan Bing ZaZhi 2016;44:833-53. 
17 Chinese Society of Cardiology of Chinese Medical Association, Editorial Board of Chinese Journal of Cardiology. Chinese Society of cardiology (CSC) guidelines for the diagnosis and management of patients with ST-segment elevation myocardial infarction. Zhong hua Xin Xue Guan Bing Za Zhi 2015;43:380-93.

18 Sterne JAC, White IR, Carlin JB, et al. Multiple imputation for missing data in epidemiological and clinical research: potential and pitfalls. BMJ 2009;338:b2393.

19 Xavier D, Pais P, Devereaux PJ, et al. Treatment and outcomes of acute coronary syndromes in India (create): a prospective analysis of registry data. Lancet 2008;371:1435-42.

20 Alexander T, Mullasari AS, Joseph G, et al. A system of care for patients with ST-segment elevation myocardial infarction in India: the Tamil Nadu-ST-Segment elevation myocardial infarction program. JAMA Cardiol 2017;2:498-505.

21 Kaifoszova Z, Kala P, Alexander T, et al. Stent for life initiative: leading example in building STEMI systems of care in emerging countries. Eurolntervention 2014;10 Suppl T:T87-95.

22 Danchin N, Popovic B, Puymirat E, et al. Five-year outcomes following timely primary percutaneous intervention, late primary percutaneous intervention, or a pharmaco-invasive strategy in STsegment elevation myocardial infarction: the FAST-MI programme. Eur Heart J 2020;41:858-66.

23 Fazel R, Joseph TI, Sankardas MA, et al. Comparison of reperfusion strategies for ST-segment-elevation myocardial infarction: a multivariate network meta-analysis. J Am Heart Assoc 2020;9:e15186

24 Mentias A, Girotra S. Pharmaco-invasive strategy: the answer to improving ST-elevation-myocardial infarction care. J Am Heart Assoc 2020;9:e16831.

25 Vora AN, Holmes DN, Rokos I, et al. Fibrinolysis use among patients requiring interhospital transfer for ST-segment elevation myocardial infarction care: a report from the US national cardiovascular data registry. JAMA Intern Med 2015;175:207-15.

26 Yin X, He Y, Zhang J, et al. Patient-level and system-level barriers associated with treatment delays for ST elevation myocardial infarction in China. Heart 2020;106:1477-82.

$27 \mathrm{Li} \mathrm{X}$, Krumholz HM, Yip W, et al. Quality of primary health care in China: challenges and recommendations. Lancet 2020;395:1802-12.
$28 \mathrm{Li} \mathrm{J,} \mathrm{Li} \mathrm{X,} \mathrm{Ross} \mathrm{JS,} \mathrm{et} \mathrm{al.} \mathrm{Fibrinolytic} \mathrm{therapy} \mathrm{in} \mathrm{hospitals} \mathrm{without}$ percutaneous coronary intervention capabilities in China from 2001 to 2011: China PEACE-retrospective AMI study. Eur Heart J Acute Cardiovasc Care 2017;6:232-43.

29 Meng Q, Mills A, Wang L, et al. What can we learn from China's health system reform? BMJ 2019;33:I2349.

30 Szummer K, Wallentin L, Lindhagen L, et al. Improved outcomes in patients with ST-elevation myocardial infarction during the last 20 years are related to implementation of evidence-based treatments: experiences from the SWEDEHEART registry 1995-2014. Eur Heart $J$ 2017;38:3056-65.

31 Peterson ED, Roe MT, Chen AY, et al. The NCDR action registryGWTG: transforming contemporary acute myocardial infarction clinical care. Heart 2010;96:1798-802.

32 Masoudi FA, Ponirakis A, de Lemos JA, et al. Trends in U.S. cardiovascular care: 2016 report from 4 ACC national cardiovascular data registries. J Am Coll Cardiol 2017;69:1427-50.

33 Puymirat E, Simon T, Cayla G, et al. Acute myocardial infarction: changes in patient characteristics, management, and 6-month outcomes over a period of 20 years in the FAST-MI program (French registry of acute ST-elevation or non-ST-elevation myocardial infarction) 1995 to 2015. Circulation 2017;136:1908-19.

34 Duber HC, McNellan CR, Wollum A, et al. Public knowledge of cardiovascular disease and response to acute cardiac events in three cities in China and India. Heart 2018;104:67-72.

35 Zhang Y, Yu B, Han Y, et al. Protocol of the China ST-segment elevation myocardial infarction (STEMI) care project (CSCAP): a 10-year project to improve quality of care by building up a regional STEMI care network. BMJ Open 2019;9:e26362.

36 Fan F, Li Y, Zhang Y, et al. Chest pain center accreditation is associated with improved in-hospital outcomes of acute myocardial infarction patients in China: findings from the CCC-ACS project. $J$ Am Heart Assoc 2019;8:e13384.

37 Sun P, Li J, Fang W, et al. Effectiveness of chest pain centre accreditation on the management of acute coronary syndrome: a retrospective study using a national database. BMJ Qual Saf $2021 ; 30: 867-75$

38 China Alliance of Chest Pain Centers. Construction plan of chest pain care units, 2020. 